\title{
The Effects of Guided Imagery on Patients Being Weaned from Mechanical Ventilation
}

\author{
LeeAnna Spiva, ${ }^{1}$ Patricia L. Hart, ${ }^{2}$ Erin Gallagher, ${ }^{3}$ Frank McVay, ${ }^{3}$ \\ Melida Garcia, ${ }^{4}$ Karen Malley, ${ }^{5}$ Marsha Kadner, ${ }^{5}$ \\ Angela Segars, ${ }^{5}$ Betsy Brakovich, ${ }^{6}$ Sonja Y. Horton, ${ }^{6}$ and Novlette Smith ${ }^{5}$ \\ ${ }^{1}$ WellStar Health System, Center for Nursing Excellence, WellStar Development Center, 2000 South Park Place, \\ Atlanta, GA 30339, USA \\ ${ }^{2}$ Kennesaw State University, 1000 Chastain Road, Prillaman Hall Building 41, Kennesaw, GA 30144, USA \\ ${ }^{3}$ WellStar Health System, Center for Nursing Excellence, 2000 South Park Place, Atlanta, GA 30339, USA \\ ${ }^{4}$ WellStar Cobb Hospital, 3950 Austell Road, Austell, GA 30106, USA \\ ${ }^{5}$ WellStar Kennestone Hospital, 677 Church Street, Marietta, GA 30060, USA \\ ${ }^{6}$ WellStar Windy Hill Hospital, 2540 Windy Hill Road, Marietta, GA 30067, USA
}

Correspondence should be addressed to LeeAnna Spiva; leeanna.spiva@wellstar.org

Received 17 June 2015; Revised 19 October 2015; Accepted 21 October 2015

Academic Editor: Tadaaki Satou

Copyright (c) 2015 LeeAnna Spiva et al. This is an open access article distributed under the Creative Commons Attribution License, which permits unrestricted use, distribution, and reproduction in any medium, provided the original work is properly cited.

\begin{abstract}
The study purpose was to assess the effects of guided imagery on sedation levels, sedative and analgesic volume consumption, and physiological responses of patients being weaned from mechanical ventilation. Forty-two patients were selected from two community acute care hospitals. One hospital served as the comparison group and provided routine care (no intervention) while the other hospital provided the guided imagery intervention. The intervention included two sessions, each lasting 60 minutes, offered during morning weaning trials from mechanical ventilation. Measurements were recorded in groups at baseline and 30- and 60minute intervals and included vital signs and Richmond Agitation-Sedation Scale (RASS) score. Sedative and analgesic medication volume consumption were recorded 24 hours prior to and after the intervention. The guided imagery group had significantly improved RASS scores and reduced sedative and analgesic volume consumption. During the second session, oxygen saturation levels significantly improved compared to the comparison group. Guided imagery group had 4.88 less days requiring mechanical ventilation and 1.4 reduction in hospital length of stay compared to the comparison group. Guided imagery may be complementary and alternative medicine (CAM) intervention to provide during mechanical ventilation weaning trials.
\end{abstract}

\section{Introduction}

Mechanical ventilation is a life-sustaining treatment for respiratory compromised patients by reducing the work to breathe, oxygenate tissue, and eliminate carbon dioxide [1]. It is a costly treatment estimated at $\$ 27$ billion a year representing $12 \%$ of hospital costs [2]. Therefore, early assessment of weaning readiness and implementation of standardized weaning trials to transition patients from full ventilator support to spontaneous breathing with the goal of early extubation $[3,4]$ are essential to prevent unintended consequences from prolonged ventilation and weaning [5]. Patients requiring mechanical ventilation may experience unintended consequences including but not limited to anxiety and inability to relax $[6,7]$, psychological and emotional distress at being unable to communicate [8], and delusional memories [7]. Analgesic and sedatives are commonly administered to reduce these symptoms. Consequently, pharmacologic interventions including sedatives and analgesics have notable side effects and are found to prolong mechanical ventilation and contribute to a higher hospital and/or intensive care unit (ICU) length of stay [9-11]. As mechanical ventilation duration increases, risk of hospital-acquired complications increases contributing to higher mortality and morbidity rates $[2,12-14]$. 
Further work is needed to explore the effects of guided imagery, a complementary and alternative medicine (CAM) technique, used to optimize a mind-body connection. Guided imagery is used to focus on pleasant mental images to promote healing and relaxation, manage symptoms, and ultimately contribute to critically ill patients' well-being [1518]. Guided imagery may have the potential to reduce the frequency and severity of symptoms in patients weaning from mechanical ventilation. Guided imagery may assist with shifting focus away from the weaning trial to acquiring a level of relaxation. Therefore, we conducted a study to determine the effects of guided imagery in mechanically ventilated patients undergoing active ventilator weaning on sedation levels, sedative and analgesic volume consumption, and physiological responses. Additionally, we assessed nurse perception of the feasibility and satisfaction of using guided imagery as an intervention.

Guided imagery has been used in a variety of patient populations including preoperative patients [19], antepartum patients [20], community-dwelling older adults [21], patients with cancer [22, 23], cardiac patients [24-26], and patients with chronic pain $[27,28]$. Patients recovering from same day head and neck surgery had a significant reduction in anxiety and pain levels and postanesthesia care unit (PACU) length of stay was nine minutes less compared to the control group [19]. There is evidence that guided imagery is an effective intervention to reduce maternal stress, fatigue, and anxiety for pregnant African American women in the second trimester [20]. Guided imagery was shown to improve selfreported leisure time behavior, reduce mobility test time, and reduce the fear of falling in older adults [21].

Researchers found lower respiratory and heart rates and blood pressure measurements and felt the sessions were beneficial for patients undergoing radiation therapy for breast cancer [23]. Similarly, thyroid patients undergoing radioactive iodine therapy had reduced fatigue and stress levels [22].

Guided imagery has been used as an intervention with cardiac patients including post-open heart surgery patients $[24,26]$ and has shown to reduce length of stay, anxiety, and pain levels. In addition, percutaneous transluminal coronary angiography patients [25] had reduced anxiety and pain levels and improved heart and respiratory rates and blood pressure after listening to a guided imagery cassette for 18 minutes.

In addition, guided imagery has been shown to reduce musculoskeletal pain and medication usage, including analgesics, in osteoarthritis patients from baseline to four months [27]. Similarly, patients with fibromyalgia who received guided imagery as an intervention had lower pain and depression levels compared to usual care [28]. However, two systematic reviews concluded that guided imagery used for musculoskeletal pain [29] and nonmusculoskeletal pain [30] were inconclusive due to lack of methodological rigor. Furthermore, the beneficial effects of guided imagery have not been studied as an intervention to provide to patients weaning from mechanical ventilation. Therefore, the specific aims of this quasi-experimental, repeated measure with intervention and comparison groups study were to assess the effects of guided imagery on patients being weaned from mechanical ventilation.

\section{Methods}

2.1. Participants. Patients were recruited from an integrated healthcare system that included two community acute care hospitals with seven ICUs located in the southeastern United States from August 1, 2012, to March 10, 2014. One hospital served as the comparison group and provided routine care (no intervention), while the other hospital provided the guided imagery intervention. The two hospitals were chosen based on similar patient population (age, reason for ICU admission, severity of illness, etc.), and evidencebased mechanical ventilation order set was followed for daily spontaneous breathing trials used to decrease practice variation. Additionally, the four researchers conducting the intervention were based at the hospital receiving the intervention. Patient inclusion criteria were (a) age greater than 18 years, (b) actively weaning from mechanical ventilation (the process of gradual reduction of ventilator support) per the hospital's standard weaning criteria, and (c) no hearing impairment. Nurse inclusion criteria included directly caring for a patient receiving the intervention. With a power of .80 , an alpha value of .05 , and a medium effect of $0.25,34$ participants were needed for the study [31, 32].

The study was reviewed and approved by Kennesaw State University Institutional Review Board and the study site's nursing research council. Informed consent was obtained at the beginning of the study by one of the study researchers from each patient's surrogate due to the patient consumption of sedatives and analgesics. In order to protect confidentiality, each participant was assigned a unique identifier.

2.2. Intervention. If patient was receiving continuous infusions of sedation and/or analgesic, the infusions were stopped in order to assess patient readiness for weaning and extubation. Prior to the intervention, patient's sedation level was assessed before weaning to ensure patient was rested, comfortable, and not lethargic, when weaning started. For patients enrolled in the guided imagery group, two separate sessions were held on two consecutive days, each lasting 60 minutes and offered during morning weaning trials. The structured, guided imagery, produced by Guided Imagery, Inc., was delivered via PLAYWAY device, $5 \times 7$ inches' plastic case. The case included a four-track preloaded 60minute audio book that required AAA battery. Disposable earphones were connected to the device. The guided imagery was narrated with a faint, soft voice, instructing the patient to relax. The session started approximately 20 minutes prior to weaning. Patients listened to the content for 60 minutes during the spontaneous breathing trial from mechanical ventilation. Four study researchers delivered the intervention and remained with the patient throughout the session. The length of time of the intervention was based on the hospital's average duration of a weaning trial (30 to 120 minutes); and the period of the delivery of the intervention was based on the weaning process occurring each morning. For patients enrolled in the comparison group, the intervention did not occur and routine patient care management was in accordance with institutional standards. Routine monitoring 
included pulse oximetry, five-lead electrocardiography, heart rate, respiratory rate, and blood pressure measurements.

2.3. Measurement. The Richmond Agitation-Sedation Scale (RASS) was developed to titrate sedation and pain control [33]. The 10-point scale ranges from unresponsive $(-5)$ to calm and alert $(0)$ to combative $(+4)$. The RASS scale has undergone extensive reliability and validity testing and is sensitive to detect changes in sedation status against level of consciousness and delirium and correlated with sedative and analgesic medication doses [34]. The RASS score is used to titrate sedation and pain control for ICU patients at the study hospitals $[33,34]$.

The Acute Physiology and Chronic Health Evaluation (APACHE II) provided an estimate of illness severity and in-hospital mortality of ICU patients. Twelve variables are used to calculate APACHE II score. Extensive reliability and validity testing has been conducted on APACHE II. The researchers conducted a retrospective chart review and recorded the worst APACHE II score during the initial 24 hours of the ICU stay [35].

The researchers developed a survey including four questions addressing feasibility and satisfaction of using guided imagery as an intervention. The survey is rated on a 5point Likert scale $(1=$ strongly disagree and $5=$ strongly agree). Nurses directly involved with patients receiving the intervention completed the surveys immediately after the intervention session. Completion of the survey by the nurse served as his or her consent to participate.

2.4. Procedures. Several times each week, the researchers communicated with the ICUs to identify potential study participants. All eligible patients were enrolled if patient met the study's inclusion criteria. Measurements were recorded by the study researchers at baseline and 30- and 60-minute intervals and included heart rate, systolic and diastolic blood pressure, respiratory rate, oxygen saturation, and RASS scores. Vital signs were measured indirectly from the noninvasive module on the monitor. Prior to data collection, the bedside monitors were tested and calibrated by the bioengineering department. Total amounts (volume) of continuous intravenous sedatives and analgesics administered in a 24-hour timeframe were converted into milliliters and recorded from the electronic documentation system by the study researchers. Sedative and analgesic amounts were evaluated during a continuous 24-hour period before and after the intervention. Commonly administered sedatives included Diprivan (propofol), dexmedetomidine (Precedex), midazolam (Versed), and lorazapam (Ativan). Commonly administered analgesics included fentanyl and morphine. The researchers reviewed the patient's medical record to collect demographic data and data to calculate APACHE II.

2.5. Data Analysis. Data were analyzed using SPSS 22.0 software for Windows (SPSS, Inc., IBM Company, Armonk, NY, USA). An independent $t$-test, Chi-square test, and MannWhitney $U$ test were conducted to examine if any differences existed between the comparison and intervention group.
A Friedman test was conducted to determine changes over time with the RASS scores and sedative volumes followed by a post hoc analysis with Wilcoxon signed-rank test with a Bonferroni correction applied. One-way repeated measures analysis of variance (ANOVA) was conducted to test the effect of guided imagery on critically ill ventilated patients' and physiological responses during both sessions at baseline, 30 minutes, and 60 minutes. To detect differences with analgesic use, $t$-tests were conducted. Significance level was set at $P<$ .05. Post hoc tests were conducted to determine where the difference in means occurred.

\section{Results}

3.1. Sample. Sample demographic characteristics are presented in Table 1. All variables between the groups were normally distributed except gender, race, RASS scores, and sedation volume. Initially, 54 patients were screened, and 42 patients receiving mechanical ventilation supported via oral endotracheal tube met study criteria and participated (Figure 1). Twenty-one patients received two 60-minute guided imagery sessions (intervention). The first session occurred within 24 hours of initial intubation and the second session followed 48 hours later. Another 21 patients served as the comparison group with no intervention and only data collection occurred within 24 hours of intubation and 48 hours later. The majority were white (69\%) females $(54.8 \%)$ with a mean age of 64.6 (SD, 13.25). Most patients were being treated with assist control (66.7\%) and primary reasons for ICU admission included respiratory (59.5\%), cardiac $(28.6 \%)$, or other $(11.9 \%)$ reasons. All patients who received the intervention were receiving one or more continuous intravenous sedative and/or analgesic infusions compared to only 16 patients in the comparison group $\left(\chi^{2}=5.76 ; P=.02\right)$. The APACHE mean score was 24.36 (SD, 7.42).

3.2. Sedation and Analgesics. The most significant effects of the intervention included improved RASS scores and a decrease in sedative and analgesic volume consumption (Table 2). During the first $\left(\chi^{2}(2)=17.45, P=.000\right)$ and second $\left(\chi^{2}(2)=7.65, P=.022\right)$ sessions there was a statistically significant difference in the RASS scores over the three time points. For the first-session median (IQR) baseline and 30-minute and 60-minute RASS scores were $-1.00(-2.00$ to 0$),-1.00(-1.25$ to 0$)$, and $-1.00(-2.00$ to 0$)$, respectively. There were significant differences between firstsession baselines and 30-minute RASS scores $(Z=-3.380$, $P=.001)$ and baseline and 60-minute RASS scores $(Z=$ $-3.252, P=.001)$. The second-session median IQR baseline and 30-minute and 60-minute RASS scores were -1.00 (-2.00 to 0$), 0(-1.00$ to 0$)$, and 0 ( -1.00 to 0$)$, respectively. There were significant differences between first-session baselines and 30minute RASS scores $(Z=-2.524, P=.012)$ and baseline and 60 -minute RASS scores $(Z=-2.480, P=.013)$. As shown in Table 2, over time the intervention group's RASS scores decreased significantly from baseline $(\mathrm{M}=-2.10)$ to 30 minutes $(\mathrm{M}=-1.57$; $\mathrm{M}$ Difference $=-.53 ; P=.01)$ and from baseline to 60 minutes $(\mathrm{M}=-1.19$; $\mathrm{M}$ Difference $=-.91$; 
TABLE 1: Demographic characteristics.

\begin{tabular}{|c|c|c|c|c|}
\hline Characteristic & $\begin{array}{c}\text { Comparison group } \\
(n=21)\end{array}$ & $\begin{array}{l}\text { Guided Imagery } \\
\quad(n=21)\end{array}$ & $\begin{array}{c}\text { Total } \\
(N=42)\end{array}$ & $P$ \\
\hline \multicolumn{5}{|l|}{ Age, $y$} \\
\hline Mean (SD) & $64(14.1)$ & $65.2(12.7)$ & $64.60(13.25)$ & .78 \\
\hline Median, range & $61,35-93$ & $66,39-89$ & $64.50,35-93$ & \\
\hline Female sex, \% & $15,71.4 \%$ & $8,38.1 \%$ & $23,54.8 \%$ & .03 \\
\hline Race, \% & & & & .05 \\
\hline White & $12,57.1 \%$ & $17,81 \%$ & $29,69 \%$ & \\
\hline African American & $7,33.3 \%$ & $1,4.8 \%$ & $8,19 \%$ & \\
\hline Others & $2,9.5 \%$ & $3,14.3 \%$ & $5,11.9 \%$ & \\
\hline Marital status, $\%$ & & & & .87 \\
\hline Married & $6,28.6 \%$ & $14,66.7 \%$ & $20,47.6 \%$ & \\
\hline Single & $6,28.6 \%$ & $2,9.55$ & $8,19 \%$ & \\
\hline Others & $9,42.9 \%$ & $5,23.8 \%$ & $14,33.3 \%$ & \\
\hline \multicolumn{5}{|l|}{ Number of comorbidities } \\
\hline Mean (SD) & $5.9(2.8)$ & $4.7(2.6)$ & $5.29(2.7)$ & .14 \\
\hline Median, range & $6,1-15$ & $4,1-10$ & $5,1-15$ & \\
\hline \multicolumn{5}{|c|}{ Acute physiology and chronic health evaluation (APACHE) score } \\
\hline Mean (SD) & $22.9(8)$ & $25.8(6.7)$ & $24.36(7.42)$ & .21 \\
\hline Median, range & $24,10-39$ & $24,9-38$ & $24,9-39$ & \\
\hline Primary reason for ICU admission, \% & & & & .09 \\
\hline Respiratory & $14,66.7 \%$ & $11,52.4 \%$ & $25,59.5 \%$ & \\
\hline Cardiac & $4,19 \%$ & $8,38.1 \%$ & $12,28.6 \%$ & \\
\hline Others & $3,14.3 \%$ & $2,9.5 \%$ & $5,11.9 \%$ & \\
\hline \multicolumn{5}{|l|}{ Hospital length of stay } \\
\hline Mean (SD) & $21.33(15.9)$ & $19.90(11.1)$ & $20.63(13.6)$ & .74 \\
\hline \multicolumn{5}{|l|}{ Total days on ventilator } \\
\hline Mean (SD) & $13.14(15.2)$ & $8.26(7.3)$ & $10.7(12.1)$ & .20 \\
\hline Median, range & $7,2-56$ & $7,1-29$ & $7,1-56$ & \\
\hline Ventilator mode & & & & .06 \\
\hline Assist control, $n \%$ & $18,85.7 \%$ & $10,47.6 \%$ & $28,66.7 \%$ & \\
\hline Synchronized intermittent mandatory, $n \%$ & $2,9.5 \%$ & $3,14.3 \%$ & $5,11.9 \%$ & \\
\hline Pressure control, $n \%$ & $1,4.8 \%$ & $8,38.1 \%$ & $9,21.4 \%$ & \\
\hline
\end{tabular}

$P=.00)$. Additionally, a significant decrease in RASS scores was noted (M Difference $=-.38 ; P=.02$ ) between the 30 minute interval $(\mathrm{M}=-1.57)$ and the 60 -minute interval $(\mathrm{M}=1.19)$. During the second session, the intervention group's RASS score decreased from baseline $(\mathrm{M}=-1.67)$ to 30 minutes $(\mathrm{M}=-1.08$; $\mathrm{M}$ Difference $=-.59)$ and from baseline to 60 minutes $(\mathrm{M}=0, \mathrm{M}$ Difference $=-1.67)$.

There was a statistically significant difference in the sedative volumes over the four time points $\left(\chi^{2}(3)=9.90\right.$, $P=.019)$. The median (IQR) 24 hours prior to (session 1 ), 24 hours after (session 1), 24 hours prior to (session 2), and 24 hours after (session 2) cumulative sedative volume totals were 145 (10 to 232 ), 42 ( 0 to 152.65 ), 3 ( 0 to 208), and 0.5 ( 0 to 115.17$)$, respectively. There were significant differences between first sessions' prior and after 24-hour cumulative volume sedative totals $(Z=-3.009, P=.003)$ and first sessions' prior and session 2 after $(Z=-2.633, P=.008)$.
The intervention group had a significant reduction in sedative volumes ( 24 hours' cumulative amount) before and after the first and second intervention sessions. The 24-hour cumulative volume was reduced by $140.06 \mathrm{~mL}$. Additionally, the intervention group had a significant reduction in analgesic volumes before and after the first intervention session $\left(t_{20}=\right.$ 2.77; $P=.01$ ) and a decrease during the second intervention but not significant (Table 2).

3.3. Physiological Responses. The intervention group mean heart rate, respiratory rate, and oxygen saturation remained well below the comparison group (Table 3). For the first intervention session only, heart rate differed significantly over the three time periods $\left(F_{2,80}=3.91 ; P=.02\right)$. Respiratory rate differed significantly over time during sessions one $\left(F_{2,80}=4.45 ; P=.02\right)$ and two $\left(F_{2,60}=3.02 ; P=\right.$ $.05)$. Both groups' heart and respiratory rates increased from 


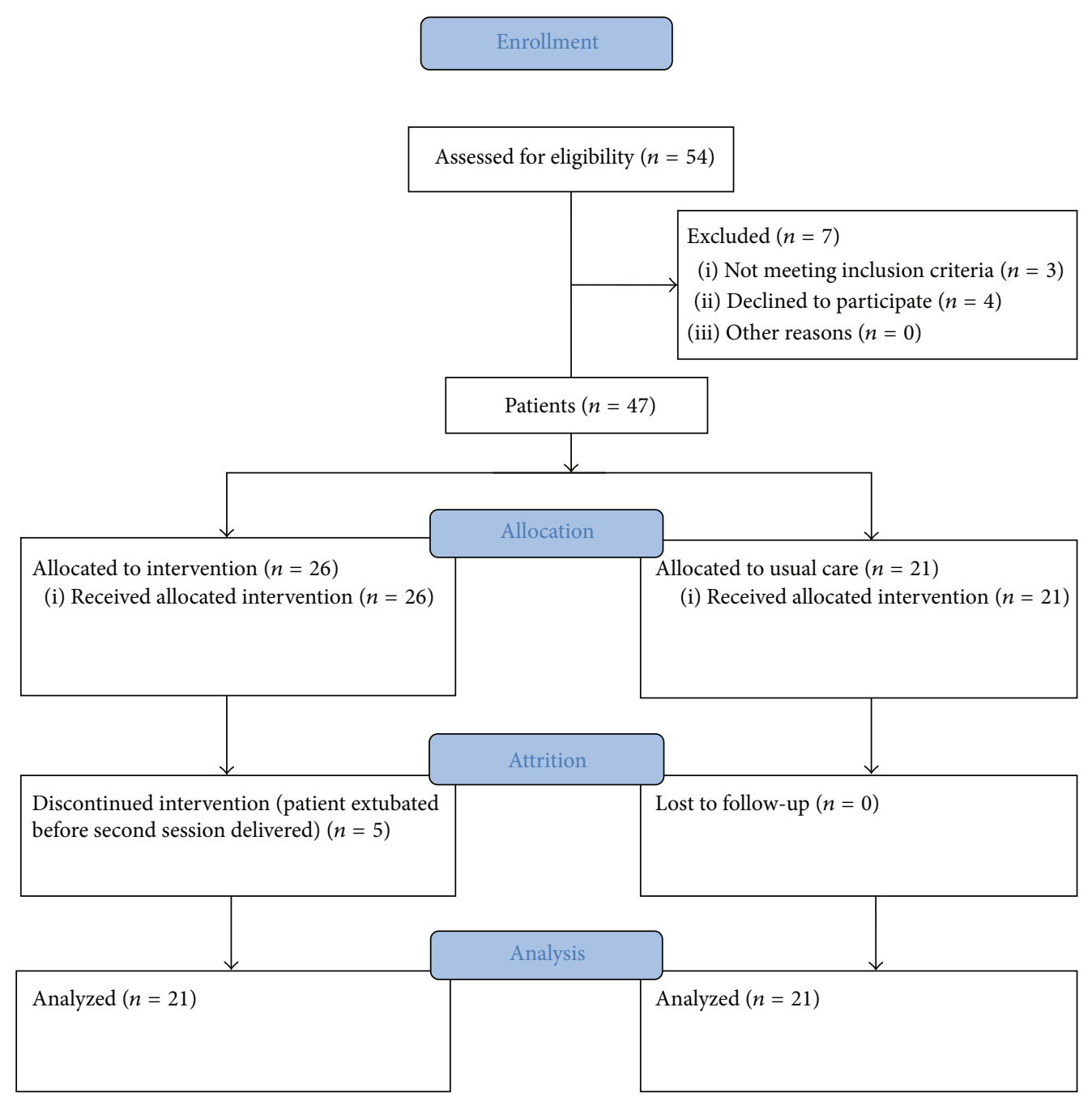

FIgURE 1: Flow diagram of patient enrollment. Adapted from Schulz K. F., Altman D. G., and Moher D. (2010). For the CONSORT Group.

baseline to 60 minutes; however, the intervention group had a lower heart and respiratory rate compared to the comparison group over the three time intervals. During the second intervention, there was a significant difference between the two groups' oxygen saturation levels $\left(F_{2,60}=3.11 ; P=.05\right)$. The intervention group had higher oxygen saturation levels during all three time periods compared to the comparison group. Furthermore, the guided imagery group had 4.88 less mechanical ventilation days compared to the comparison group $\left(t_{39}=1.33 ; P=.193\right)$. The guided imagery group hospital length of stay was 1.4 less days compared to the comparison group $\left(t_{40}=.33 ; P=.74\right)$.

3.4. Staff Perception. Of the 42 surveys that were distributed, 23 nurses (55\%) completed the survey with mean scores for each question ranging from 4.09 to 4.83 . Nurses felt that guided imagery was an effective nursing intervention $(\mathrm{M}=4.83, \mathrm{SD}=.39)$. Nurses felt that the intervention was successfully incorporated into the weaning process $(M=4.09$, $\mathrm{SD}=.95)$ and simple to implement $(\mathrm{M}=4.13, \mathrm{SD}=1.0)$ and the intervention met the intended purpose $(\mathrm{M}=4.09, \mathrm{SD}=$ 1.08).

\section{Discussion}

To date, research conducted has focused on deployment of interventions with no research identified using guided imagery as an intervention in patients who are being actively weaned from the ventilator. This study is unique in using guided imagery as an intervention in mechanically ventilated patients who were being actively weaned from the ventilator. Despite the intervention group having higher RASS scores and receiving continuous sedative and analgesic infusions, we demonstrated improved RASS scores, reduced sedative and analgesic volume consumption, and higher oxygen saturation levels. Furthermore, we found that patients who received the intervention had a shorter time on the ventilator and shorter length of stay. We were able to demonstrate a significant improvement in actual sedative and analgesic volume intake in relation to using guided imagery as an intervention despite $24 \%$ of the interventions groups' baseline RASS score being 
TABLE 2: Sedation levels, sedative, and analgesic outcomes.

\begin{tabular}{|c|c|c|c|}
\hline Characteristic & $\begin{array}{l}\text { Comparison group } \\
(n=21), \text { mean }(\mathrm{SD})\end{array}$ & $\begin{array}{c}\text { Guided imagery } \\
(n=21), \text { mean }(\mathrm{SD})\end{array}$ & $P$ \\
\hline \multicolumn{4}{|l|}{ Session I } \\
\hline Baseline RASS score & $-.38(1.1)$ & $-2.10(1.4)$ & .000 \\
\hline 30-minute RASS score & $0.1(1.2)$ & $-1.57(1.4)$ & .000 \\
\hline 60-minute RASS score & $-.29(1.2)$ & $-1.19(1.8)$ & .086 \\
\hline 24-hour cumulative amount $(\mathrm{mL})$ of sedative infused before & $\begin{array}{l}133.18 \text { (143.28) } \\
\quad(16 \text { doses })\end{array}$ & $\begin{array}{l}218.66(243.62) \\
\quad(19 \text { doses })\end{array}$ & .284 \\
\hline 24-hour cumulative amount $(\mathrm{mL})$ of analgesic infused before & $\begin{array}{l}48.92(83.77) \\
\quad(11 \text { doses })\end{array}$ & $\begin{array}{l}95.28(128.12) \\
\quad(14 \text { doses })\end{array}$ & .173 \\
\hline 24-hour cumulative amount $(\mathrm{mL})$ of sedative infused after & $\begin{array}{l}111.77 \text { (156.24) } \\
\quad(13 \text { doses })\end{array}$ & $\begin{array}{l}78.59 \text { (93.61) } \\
(13 \text { doses })\end{array}$ & .737 \\
\hline 24-hour cumulative amount $(\mathrm{mL})$ of analgesic infused after & $\begin{array}{l}54.69(89.28) \\
\quad(11 \text { doses })\end{array}$ & $\begin{array}{l}18.10(51.36) \\
\quad(8 \text { doses })\end{array}$ & .111 \\
\hline \multicolumn{4}{|l|}{ Session II } \\
\hline Baseline RASS score & $-.55(.8)$ & $-1.67(2)$ & .043 \\
\hline 30-minute RASS score & $-.35(.9)$ & $-1.08(1.8)$ & .162 \\
\hline 60-minute RASS score & $-0.55(.8)$ & $0(1.6)$ & .181 \\
\hline 24-hour cumulative amount $(\mathrm{mL})$ of sedative infused before & $\begin{array}{l}109.24 \text { (146.74) } \\
\quad(14 \text { doses })\end{array}$ & $\begin{array}{l}166.54(259.61) \\
\quad(10 \text { doses })\end{array}$ & .803 \\
\hline 24-hour cumulative amount $(\mathrm{mL})$ of analgesic infused before & $\begin{array}{l}24 \text { (52.67) } \\
\text { (6 doses) }\end{array}$ & $\begin{array}{l}2.52(9.13) \\
(4 \text { doses })\end{array}$ & .073 \\
\hline 24-hour cumulative amount $(\mathrm{mL})$ of sedative infused after & $\begin{array}{l}125.93 \text { (188.45) } \\
\quad(15 \text { doses })\end{array}$ & $\begin{array}{l}38.13 \text { (75.78) } \\
\text { (3 doses })\end{array}$ & .024 \\
\hline 24-hour cumulative amount $(\mathrm{mL})$ of analgesic infused after & $\begin{array}{l}21.14 \text { (56.36) } \\
(4 \text { doses })\end{array}$ & $\begin{array}{l}.49(1.53) \\
(3 \text { doses })\end{array}$ & .101 \\
\hline
\end{tabular}

greater than or equal to minus four $(-4)$ indicating that the patient was deeply sedated.

Throughout the intervention, heart rate, diastolic blood pressure, and oxygen saturation levels remained within normal range. Similar to other researcher findings that used guided imagery, we found that heart and respiratory rates were significantly lower over time for the intervention group compared to the comparison group $[23,25]$. The comparison group's respiratory rate increased and oxygen consumption declined. We did not find significant improvements in blood pressure but other medications including cardiac medications could have masked the intervention effects. Similar to Deisch et al. [24] and Halpin et al. [26] patients who received the guided imagery intervention had reduced length of hospital stay (1.4 less days) and 4.88 less mechanical ventilation days compared to the comparison group.

Complementary and alternative medicine therapy such as guided imagery may be a part of the multimodal treatment approach and serve as a substitute to administering high doses of sedatives to assist with keeping the patient calm and relaxed. Nurses perceived the intervention as effective and easily incorporated into the weaning process.

Our study had several limitations. The sample was primarily white females admitted to ICU with a respiratory problem. Sedation levels and sedative and analgesic use and practices may have varied between the hospitals and affected measurements. We only looked at volumes of sedatives and analgesics, as most of these medications are weight based; comparing volume of medications infused between groups is a limitation. Additionally, before intervention the intervention group had higher cumulative amounts of sedative and analgesics that might have influenced the amount of sedatives and analgesics needed during and after the intervention. Both hospitals' ventilator weaning is assumed by the respiratory therapist guided by standardized protocols. Daily weaning occurred in the mornings and intervention effects may have been different later in the day. Certain medications may have masked the intervention effects as we did not control for prescribed medications such as cardiac medications. Secondary to one hospital serving as the intervention hospital and the other serving as the control hospital, any hospital effect is potentially confounded by the intervention effect. By carrying out the intervention at one hospital only, the researchers were hoping to lessen the threat of treatment diffusion. It is difficult to be blinded to the intervention when the researchers had to deliver the intervention to the patient. A randomized controlled trial (RCT) was not conducted secondary to conditions that either occurred daily and/or were planned in the ICU that the researchers had no control over things including but not limited to noise levels, patient volumes, ICU renovations, and transition from one electronic medical record to another which would have added 
TABLE 3: Physiological outcomes.

\begin{tabular}{|c|c|c|c|c|}
\hline Characteristic & $\begin{array}{l}\text { Comparison group } \\
(n=21)\end{array}$ & $\begin{array}{l}\text { Guided imagery } \\
\quad(n=21)\end{array}$ & $\begin{array}{c}\text { Total } \\
(N=42)\end{array}$ & $P$ \\
\hline \multicolumn{5}{|l|}{ Session I } \\
\hline \multicolumn{5}{|l|}{ Baseline, mean (SD) } \\
\hline Heart rate & $84.7(16.8)$ & $79.1(19)$ & $81.9(17.7)$ & .317 \\
\hline Systolic blood pressure (BP) & $124.7(22.9)$ & $126.6(19)$ & $125.6(21)$ & .777 \\
\hline Diastolic BP & $59.3(11.8)$ & $64.1(17.4)$ & $61.7(17.9)$ & .305 \\
\hline Respiratory rate & $21.8(4.5)$ & $19.8(4.8)$ & $20.8(4.7)$ & .170 \\
\hline Oxygen saturation & $97.6(2.5)$ & $97.6(2.4)$ & $97.6(2.4)$ & 1.000 \\
\hline \multicolumn{5}{|l|}{30 minutes, mean (SD) } \\
\hline Heart rate & $89.3(22.2)$ & $81.6(18)$ & $85.5(20.3)$ & .220 \\
\hline Systolic BP & $133.9(17.4)$ & $127.2(20)$ & $130.5(19)$ & .258 \\
\hline Diastolic BP & $64.5(12.2)$ & $64.7(16.1)$ & $64.6(14.1)$ & .966 \\
\hline Respiratory rate & $22.6(4)$ & $20.3(6.8)$ & $21.5(5.7)$ & .186 \\
\hline Oxygen saturation & $97.1(2.7)$ & $97.2(3)$ & $97.1(2.8)$ & .915 \\
\hline \multicolumn{5}{|l|}{60 minutes, mean $(S D)$} \\
\hline Heart rate & $90.3(16.4)$ & $82.7(16)$ & $86.5(16.3)$ & .130 \\
\hline Systolic BP & $129.6(24.4)$ & $130.2(25)$ & $129.9(24.3)$ & .940 \\
\hline Diastolic BP & $62(11.9)$ & $63.8(20)$ & $62.9(16.2)$ & .728 \\
\hline Respiratory rate & $24.6(4.7)$ & $21.6(8.1)$ & $23.1(6.7)$ & .148 \\
\hline Oxygen saturation & $97.2(2.5)$ & $97.1(2.8)$ & $97.1(2.6)$ & .818 \\
\hline \multicolumn{5}{|l|}{ Session II } \\
\hline \multicolumn{5}{|l|}{ Baseline, mean (SD) } \\
\hline Heart rate & $86.5(17.4)$ & $77.4(14.8)$ & $83.1(16.8)$ & .141 \\
\hline Systolic BP & $123.8(25.8)$ & $133.9(20)$ & $127.6(24.1)$ & .254 \\
\hline Diastolic BP & $60(12.3)$ & $65.8(21.3)$ & $62.2(16.2)$ & .338 \\
\hline Respiratory rate & $23.4(3.6)$ & $20.9(4.6)$ & $22.5(4.1)$ & .099 \\
\hline Oxygen saturation & $96.6(3)$ & $96.9(2.2)$ & $96.7(2.7)$ & .733 \\
\hline \multicolumn{5}{|l|}{30 minutes, mean (SD) } \\
\hline Heart rate & $94(14.7)$ & $82.8(19.4)$ & $89.8(17.2)$ & .073 \\
\hline Systolic BP & $141.4(25.8)$ & $135.8(13.7)$ & $139.3(22)$ & .622 \\
\hline Diastolic BP & $63.3(10.8)$ & $66.7(17.7)$ & $64.6(13.6)$ & .426 \\
\hline Respiratory rate & $22.2(4.8)$ & $23.8(7)$ & $22.8(5.7)$ & .507 \\
\hline Oxygen saturation & $96.9(2.8)$ & $97.2(2.3)$ & $97(2.6)$ & .427 \\
\hline \multicolumn{5}{|l|}{60 minutes, mean $(\mathrm{SD})$} \\
\hline Heart rate & $92.4(21.2)$ & $81.5(12.5)$ & $88.3(19)$ & .119 \\
\hline Systolic BP & $124.6(21.7)$ & $136.9(21.7)$ & $129.2(22.2)$ & .130 \\
\hline Diastolic BP & $62.5(14.9)$ & $68.7(15.6)$ & $64.8(15.2)$ & .271 \\
\hline Respiratory rate & $23.3(5.5)$ & $20.8(5.6)$ & $22.4(5.6)$ & .235 \\
\hline Oxygen saturation & $95.6(3.8)$ & $97.9(1.6)$ & $96.5(3.3)$ & .024 \\
\hline
\end{tabular}

to additional study limitations. It is suggested to replicate the study randomizing the intervention at both hospitals to see if the findings of the present study are generalizable.

As we noted, weaning trials and intervention sessions occurred during the morning hours. We attempted to obtain surrogates' perceptions of ventilated patients who listened to the guided imagery. We did not capture enough data for analysis primarily due to low participation. Typically, the patients' surrogate consented for the patient to participate the day prior to the actual intervention and/or the surrogate was not present during the entire weaning process and intervention not meeting study criteria. Future investigators may want to involve patients' surrogate in intervention and weaning process to promote patient- and family-centered care. In addition, patients' surrogates were not always present at the hospital with the patient and initial weaning trial was unpredictable which at times made recruitment and data collection a challenge for the researchers. ICU nurses' 
perception of guided imagery as an effective intervention to implement was rather high; however, the four researchers delivering the intervention may have inadvertently positively skewed the nurses' perception of guided imagery. Furthermore, the intervention effect sustained beyond the study time is unknown.

\section{Conclusions}

Guided imagery may be a CAM intervention to provide during mechanical ventilation weaning trials. Guided imagery appeared to be effective, safe, and feasible intervention to use in patients being weaned from mechanical ventilation. Future research is needed including a larger randomized controlled trial examining the effect of guided imagery use with a larger sample with a longer tracking period in relation to patient outcomes.

\section{Disclosure}

Institution work was performed in WellStar Health System Kennestone and Cobb Hospitals.

\section{Conflict of Interests}

The authors declare that there is no conflict of interests regarding the publication of this paper.

\section{Funding}

The researchers received $\$ 150$ to purchase the music and guided imagery devices to use in this study through WellStar's Nursing Research Fund. The researchers also received a discounted price for the PLAYWAY device at Findaway World.

\section{Acknowledgments}

The researchers acknowledge Amy Good, BSN, RN for her assistance with data collection.

\section{References}

[1] L. D. Urden, K. M. Stacy, and M. E. Lough, Critical Care Nursing: Diagnosis and Management, Elsevier Mosby, St. Louis, Mo, USA, 7th edition, 2014.

[2] H. Wunsch, W. T. Linde-Zwirble, D. C. Angus, M. E. Hartman, E. B. Milbrandt, and J. M. Kahn, "The epidemiology of mechanical ventilation use in the United States," Critical Care Medicine, vol. 38, no. 10, pp. 1947-1953, 2010.

[3] B. Blackwood, F. Alderdice, K. Burns, C. Cardwell, G. Lavery, and P. O'Halloran, "Use of weaning protocols for reducing duration of mechanical ventilation in critically ill adult patients: cochrane systematic review and meta-analysis," The British Medical Journal, vol. 342, Article ID c7237, 2011.

[4] B. Blackwood, K. E. A. Burns, C. R. Cardwell, and P. O'Halloran, "Protocolized versus non-protocolized weaning for reducing the duration of mechanical ventilation in critically ill adult patients," Cochrane Database of Systematic Reviews, vol. 11, Article ID CD006904, 2014.

[5] American Association of Critical-Care Nurses (AACN), "AACN PEARL: Implementing the ABCDE bundle at the bedside," 2013, http://www.aacn.org/wd/practice/content/actionpak/ withlinks-abcde-toolkit.pcms?menu=practice.

[6] L. L. Chlan, C. R. Weinert, A. Heiderscheit et al., "Effects of patient-directed music intervention on anxiety and sedative exposure in critically Ill patients receiving mechanical ventilatory support: a randomized clinical trial," The Journal of the American Medical Association, vol. 309, no. 22, pp. 2335-2344, 2013.

[7] L. Rose, M. Nonoyama, S. Rezaie, and I. Fraser, "Psychological wellbeing, health related quality of life and memories of intensive care and a specialised weaning centre reported by survivors of prolonged mechanical ventilation," Intensive and Critical Care Nursing, vol. 30, no. 3, pp. 145-151, 2014.

[8] R. Khalaila, W. Zbidat, K. Anwar, A. Bayya, D. M. Linton, and S. Sviri, "Communication difficulties and psychoemotional distress in patients receiving mechanical ventilation," American Journal of Critical Care, vol. 20, no. 6, pp. 470-479, 2011.

[9] C. R. Dale, D. A. Kannas, V. S. Fan et al., "Improved analgesia, sedation, and delirium protocol associated with decreased duration of delirium and mechanical ventilation," Annals of the American Thoracic Society, vol. 11, no. 3, pp. 367-374, 2014.

[10] T. D. Girard, J. P. Kress, B. D. Fuchs et al., "Efficacy and safety of a paired sedation and ventilator weaning protocol for mechanically ventilated patients in intensive care (Awakening and Breathing Controlled trial): a randomised controlled trial," The Lancet, vol. 371, no. 9607, pp. 126-134, 2008.

[11] M. J. Grap, C. L. Munro, P. A. Wetzel et al., "Sedation in adults receiving mechanical ventilation: physiological and comfort outcomes," American Journal of Critical Care, vol. 21, no. 3, pp. e53-e64, 2012.

[12] Y. Hayashi, K. Morisawa, M. Klompas et al., "Toward improved surveillance: the impact of ventilator-associated complications on length of stay and antibiotic use in patients in intensive care units," Clinical Infectious Diseases, vol. 56, no. 4, pp. 471-477, 2013.

[13] A. C. Lemay, A. Anzueto, M. I. Restrepo, and E. M. Mortensen, "Predictors of long-term mortality after severe sepsis in the elderly," American Journal of the Medical Sciences, vol. 347, no. 4, pp. 282-288, 2014.

[14] D. M. Needham, E. Colantuoni, P. A. Mendez-Tellez et al., "Lung protective mechanical ventilation and two year survival in patients with acute lung injury: prospective cohort study," The British Medical Journal, vol. 344, no. 7854, Article ID e2124, 2012.

[15] M. S. Micozzi and A. Michael, Fundamentals of Complementary and Alternative Medicine, Saunders Elsevier, St. Louis, Mo, USA, 5 th edition, 2015.

[16] National Center for Complementary and Integrative Health, "Relaxation techniques forhealth," 2014, https://nccih.nih.gov/ sites/nccam.nih.gov/files/Get_The_Facts_Relaxation_Techniques_ 02-06-2015.pdf.

[17] D. Kramlich, "Introduction to complementary, alternative, and traditional therapies," Critical Care Nurse, vol. 34, no. 6, pp. 5056, 2014.

[18] M. F. Tracy and L. Chlan, "Nonpharmacological interventions to manage common symptoms in patients receiving mechanical ventilation," Critical Care Nurse, vol. 31, no. 3, pp. 19-28, 2011. 
[19] E. A. Gonzales, R. J. A. Ledesma, D. J. McAllister, S. M. Perry, C. A. Dyer, and J. P. Maye, "Effects of guided imagery on postoperative outcomes in patients undergoing same-day surgical procedures: a randomized, single-blind study," AANA Journal, vol. 78, no. 3, pp. 181-188, 2010.

[20] N. Jallo, R. J. Ruiz, R. K. Elswick, and E. French, "Guided imagery for stress and symptom management in pregnant African American women," Evidence-based Complementary and Alternative Medicine, vol. 2014, Article ID 840923, 13 pages, 2014.

[21] B. H. Kim, R. A. Newton, M. L. Sachs, J. J. Glutting, and K. Glanz, "Effect of guided relaxation and imagery on falls selfefficacy: a randomized controlled trial," Journal of the American Geriatrics Society, vol. 60, no. 6, pp. 1109-1114, 2012.

[22] M. H. Lee, D.-H. Kim, and H. S. Yu, "The effect of guided imagery on stress and fatigue in patients with thyroid cancer undergoing radioactive iodine therapy," Evidence-based Complementary and Alternative Medicine, vol. 2013, Article ID 130324, 8 pages, 2013.

[23] D. Serra, C. R. Parris, E. Carper et al., "Outcomes of guided imagery in patients receiving radiation therapy for breast cancer," Clinical Journal of Oncology Nursing, vol. 16, no. 6, pp. 617-623, 2012.

[24] P. Deisch, S. M. Soukup, P. Adams, and M. C. Wild, "Guided imagery: replication study using coronary artery bypass graft patients," Nursing Clinics of North America, vol. 35, no. 2, pp. 417-425, 2000.

[25] S. Foji, M. A. Tadayonfar, M. Mohsenpour, and M. H. Rakhshani, "The study of the effect of guided imagery on pain, anxiety and some other hemodynamic factors in patients undergoing coronary angiography," Complementary Therapies in Clinical Practice, vol. 21, no. 2, pp. 119-123, 2015.

[26] L. S. Halpin, A. M. Speir, P. CapoBianco, and S. D. Barnett, "Guided imagery in cardiac surgery," Outcomes Management, vol. 6, no. 3, pp. 132-137, 2002.

[27] C. L. Baird, M. M. Murawski, and J. Wu, "Efficacy of guided imagery with relaxation for osteoarthritis symptoms and medication intak," Pain Management Nursing, vol. 11, no. 1, pp. 56-65, 2010.

[28] M. D. Onieva-Zafra, L. H. M. García, and M. G. Del Valle, "Effectiveness of guided imagery relaxation on levels of pain and depression in patients diagnosed with fibromyalgia," Holistic Nursing Practice, vol. 29, no. 1, pp. 13-21, 2015.

[29] P. Posadzki and E. Ernst, "Guided imagery for musculoskeletal pain: a systematic review," Clinical Journal of Pain, vol. 27, no. 7, pp. 648-653, 2011.

[30] P. Posadzki, W. Lewandowski, R. Terry, E. Ernst, and A. Stearns, "Guided imagery for non-musculoskeletal pain: a systematic review of randomized clinical trials," Journal of Pain and Symptom Management, vol. 44, no. 1, pp. 95-104, 2012.

[31] F. Faul, E. Erdfelder, A.-G. Lang, and A. Buchner, "G* Power 3: a flexible statistical power analysis program for the social, behavioral, and biomedical sciences," Behavior Research Methods, vol. 39, no. 2, pp. 175-191, 2007.

[32] F. Faul, E. Erdfelder, A. Buchner, and A.-G. Lang, "Statistical power analyses using $\mathrm{G}^{*}$ Power 3.1: tests for correlation and regression analyses," Behavior Research Methods, vol. 41, no. 4, pp. 1149-1160, 2009.

[33] C. N. Sessler, M. S. Gosnell, M. J. Grap et al., "The richmond agitation-sedation scale: validity and reliability in adult intensive care unit patients," American Journal of Respiratory and Critical Care Medicine, vol. 166, no. 10, pp. 1338-1344, 2002.
[34] E. W. Ely, B. Truman, A. Shintani et al., "Monitoring sedation status over time in ICU patients: reliability and validity of the Richmond Agitation-Sedation Scale (RASS)," The Journal of the American Medical Association, vol. 289, no. 22, pp. 2983-2991, 2003.

[35] W. A. Knaus, E. A. Draper, D. P. Wagner, and J. E. Zimmerman, "APACHE II: a severity of disease classification system," Critical Care Medicine, vol. 13, no. 10, pp. 818-829, 1985. 


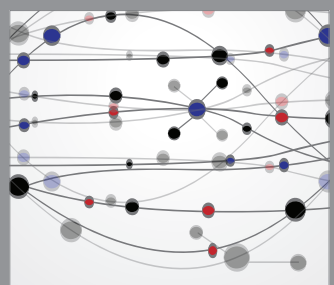

The Scientific World Journal
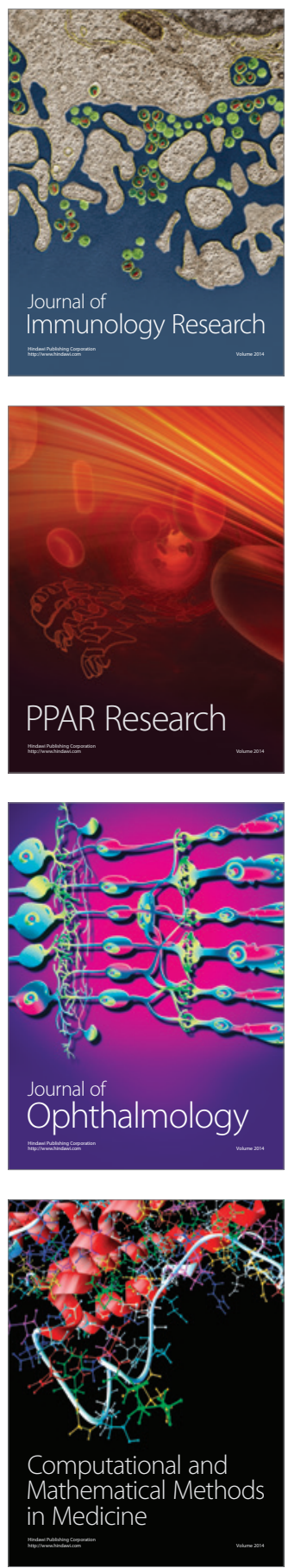

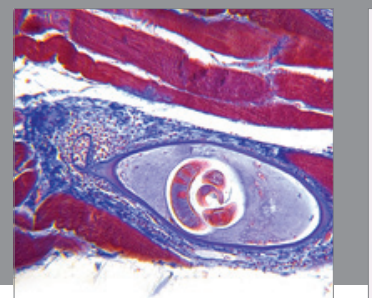

Gastroenterology

Research and Practice
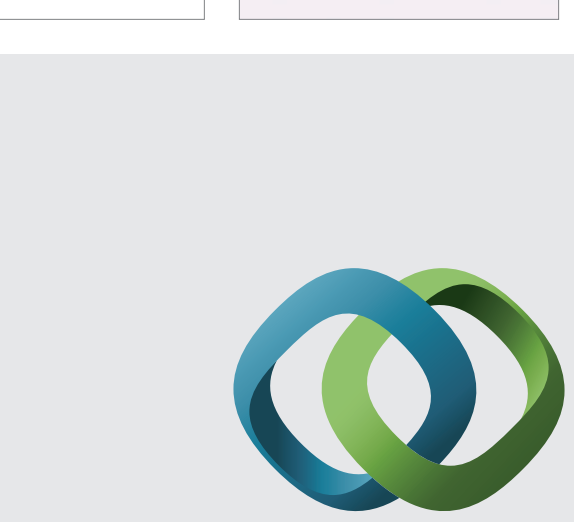

\section{Hindawi}

Submit your manuscripts at

http://www.hindawi.com
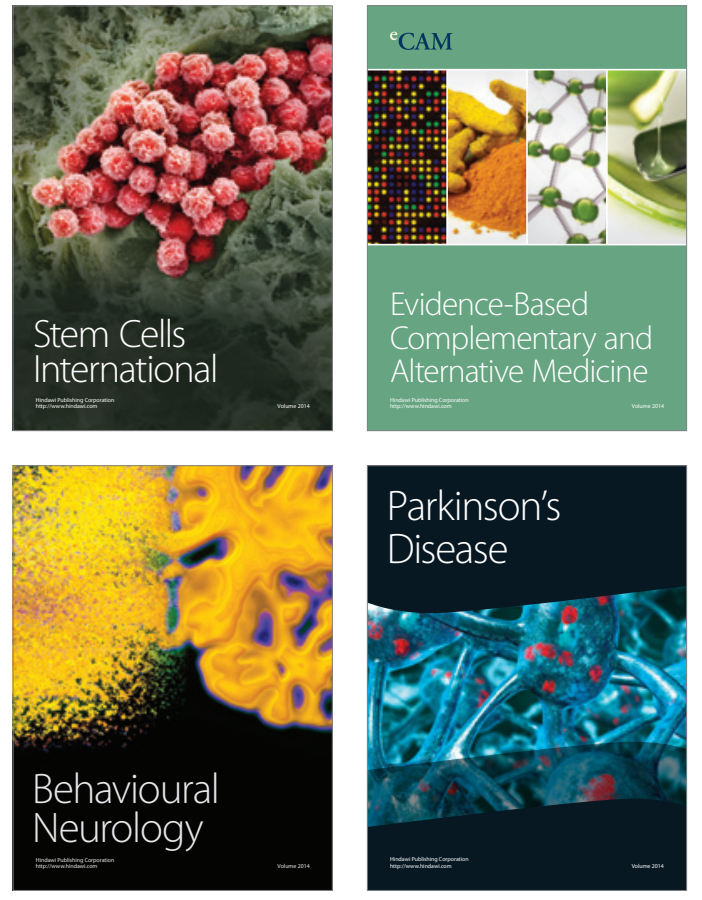
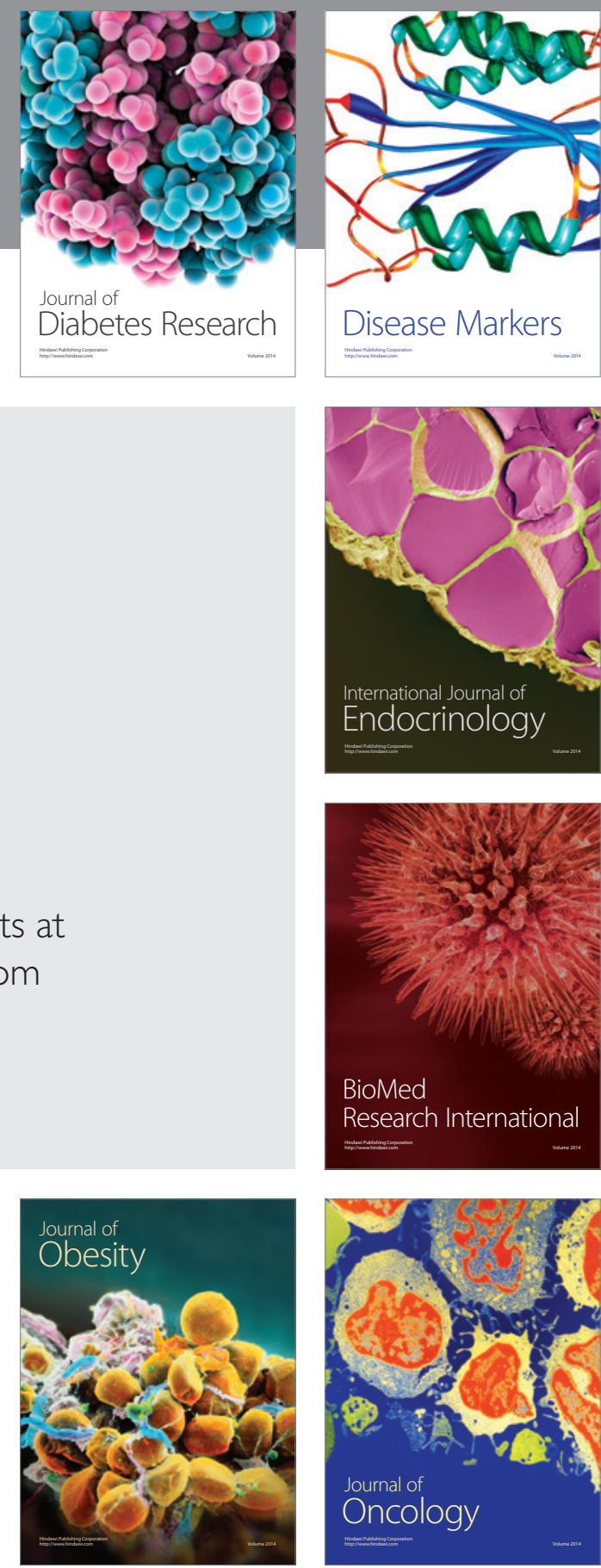

Disease Markers
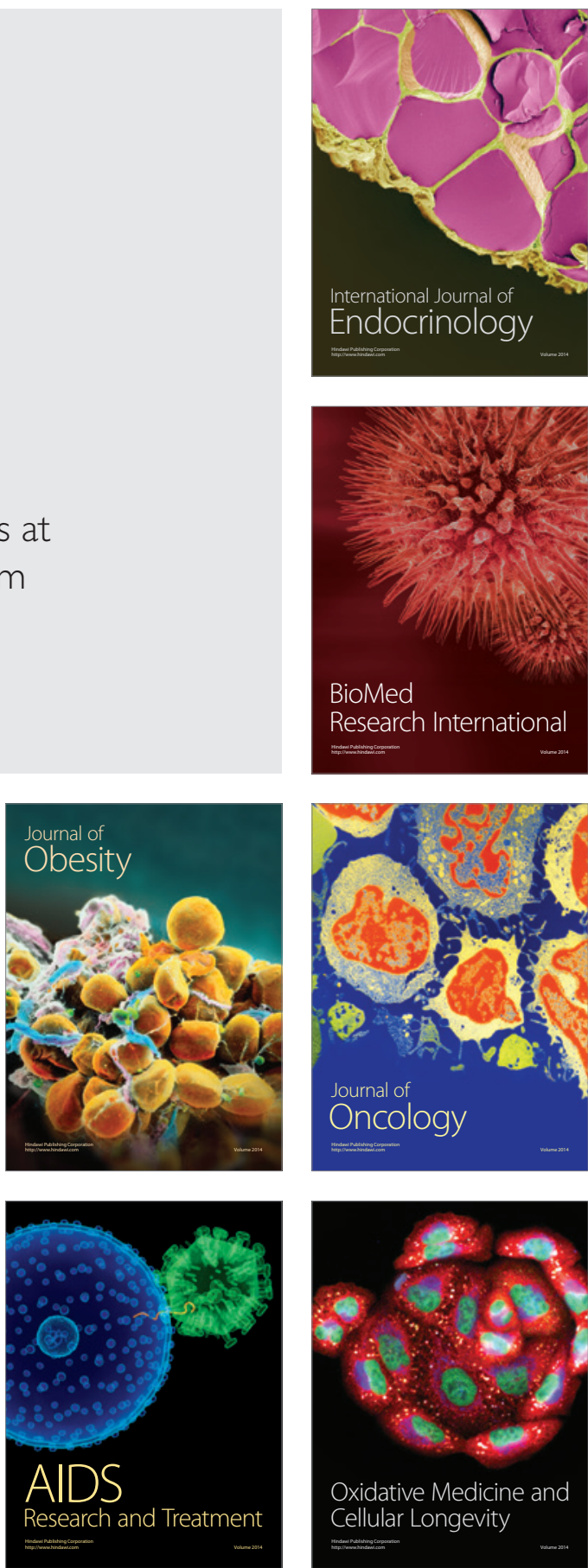